Check for updates

Cite this: Mol. Omics, 2019, 15,247

DOI: $10.1039 / c 9 m o 90005 e$

rsc.li/molomics

\section{Correction: Functional specific roles of FADD: comparative proteomic analyses from knockout cell lines}

\author{
Hongqin Zhuang, ${ }^{a}$ Ziyi Gan, ${ }^{a}$ Weiwei Jiang, ${ }^{a}$ Xiangyu Zhang ${ }^{a}$ and Zi-Chun Hua*ab
}

Correction for 'Functional specific roles of FADD: comparative proteomic analyses from knockout cell lines' by Hongqin Zhuang et al., Mol. BioSyst., 2013, 9, 2063-2078.

The authors wish to draw the reader's attention to their closely related paper, published in Proteomics, ${ }^{1}$ which should have been cited in this Molecular BioSystems paper.

In both papers, the authors used proteomics analysis to investigate two different status of the FADD protein (FADD KO and FADD-phosphorylation) and explore their distinct participation in cell signalling. In this Molecular BioSystems paper, the authors found that FADD KO-induced differentially-expressed proteins were involved in lipid metabolism. Bioinformatic analyses revealed HNF4-alpha, c-Myc, SP1, YB-1, C/EBP and AP1 as several possible key transcriptional factors lying at the center of dysregulated pathways. In ref. 1, the authors found that FADD phosphorylation-induced differentially-expressed proteins were involved in lipid metabolism and glycolysis.

The rank of process networks clustered with the differentially-expressed proteins was different in response to FADD KO and FADD phosphorylation, indicating their different contribution to cell signalling. Through these two papers, the authors found that FADD might be involved in lipid metabolism and glucose metabolism, and FADD KO and FADD phosphorylation have similar effects in several signalling pathways, while in some signalling pathways their effects are different.

GeneGo disease biomarker networks analysis demonstrated the relevance between the differentially-expressed proteins by FADD deficiency and obesity (this paper), while GeneGo Diseases enrichment analysis revealed the possible functions of FADD phosphorylation in mitochondrial diseases and glucose metabolism disorders (ref. 1).

The authors apologise for duplicating two figures, which were used as wild-type controls for 2D gel images and transmission electron microscopy, in Fig. 1A and 10A in this paper and Fig. 1A and 5A in ref. 1.

In addition, the authors regret that there is unattributed overlap in text between this Molecular BioSystems paper and ref. 1.

The Royal Society of Chemistry apologises for these errors and any consequent inconvenience to authors and readers.

\title{
References
}

1 H. Zhuang, Z. Gan, W. Jiang, X. Zhang and Z.-C. Hua, Proteomics, 2013, 13, 2398-2413.

\footnotetext{
${ }^{a}$ The State Key Laboratory of Pharmaceutical Biotechnology, College of Life Science and School of Stomatology, Affiliated Stomatological Hospital, Nanjing University, Nanjing 210093, Jiangsu, P. R. China. E-mail: hzc1117@nju.edu.cn; Fax: +86 25-83324605; Tel: +86 25-83593692

${ }^{b}$ Changzhou High-Tech Research Institute of Nanjing University and Jiangsu TargetPharma Laboratories Inc., Changzhou 213164, Jiangsu, P. R. China
} 